\title{
High-order aberration changes after femtosecond LASIK surgery in patients with high myopia
}

\author{
Ziqing Feng ${ }^{1 \wedge}$, Qianru Wang ${ }^{2}$, Chunyu $\mathrm{Du}^{2}$, Fan Yang ${ }^{2},{\mathrm{Xue} \mathrm{Li}^{2} \wedge}^{\wedge}$ \\ ${ }^{1}$ Zhongshan Ophthalmic Center, State Key Laboratory of Ophthalmology, Sun Yat-sen University, Guangzhou, China; ${ }^{2}$ Department of \\ Ophthalmology, Center of Optometry of the First Affiliated Hospital of Harbin Medical University, Harbin, China \\ Contributions: (I) Conception and design: Z Feng, X Li; (II) Administrative support: X Li; (III) Provision of study materials or patients: X Li; (IV) \\ Collection and assembly of data: Z Feng, Q Wang, C Du, F Yang; (V) Data analysis and interpretation: Z Feng, Q Wang; (VI) Manuscript writing: \\ All authors; (VII) Final approval of manuscript: All authors. \\ Correspondence to: Xue Li. Department of Ophthalmology, Center of Optometry of the First Affiliated Hospital of Harbin Medical University, Harbin \\ 150001, China. Email: 13351282838@163.com.
}

\begin{abstract}
Background To investigate the relationship between high-order aberration (HOA) changes and RCST/CT (residual corneal stroma thickness/corneal thickness) after femtosecond LASIK surgery.

Methods: A total of 65 eyes from 39 patients with high myopia, who had femtosecond (FS) laser-assisted in situ keratomileusis (LASIK) surgery performed in our hospital, were included in this study. HOA and central corneal thickness were measured preoperatively and at 1 week, 1 month, and 3 months after FS-LASIK by Sirius Scheimpflug-Placido topography. Residual corneal stroma thickness (RCST) and ablation depth were measured during surgery.
\end{abstract}

Results: Horizontal coma $\left(\mathrm{Z}_{3}{ }^{1}\right)$, spherical aberration $\left(\mathrm{Z}_{4}{ }^{0}\right)$, second horizontal coma $\left(\mathrm{Z}_{5}{ }^{1}\right)$, second horizontal trefoil $\left(Z_{5}^{3}\right)$, pentafoil $\left(Z_{5}^{5}\right)$, second spherical aberration $\left(Z_{6}{ }^{0}\right)$, and total HOAs were significantly increased at 1 week, 1 month, and 3 months after surgery compared with the preoperative values $(\mathrm{P}<0.05)$, but no significant differences were found in horizontal trefoil $\left(\mathrm{Z}_{3}{ }^{3} ; \mathrm{P}>0.05\right)$. Furthermore, $\mathrm{Z}_{3}{ }^{1}, \mathrm{Z}_{4}{ }^{0}$, and HOAs were significantly increased at 3 months post-surgery compared with the values at 1 week post-operation $(\mathrm{P}<0.05)$. Positive correlations were found between ablation depth and $Z_{6}{ }^{0}$ at 1 week and 1 month after surgery $(r=0.291$ and 0.337 , respectively; $\mathrm{P}<0.05$ ). Ablation depth was positively correlated with $Z_{4}{ }^{0}$ and $Z_{6}{ }^{0}$ at 3 months after surgery ( $\mathrm{r}=0.439$ and 0.336 , respectively; $\mathrm{P}<0.05$ ). The RCST/CT was negatively correlated with $\mathrm{Z}_{4}^{0}, \mathrm{Z}_{6}{ }^{0}$, and HOAs at 3 months after surgery $(r=-0.322,-0.412$, and -0.321 , respectively; $\mathrm{P}<0.05)$. There were no significant correlations between Zernike coefficients in terms of high-order aberration, HOAs, and RCST/ CT at 1 week and 1 month post-surgery.

Conclusions: Corneal HOAs in high myopia patients increased significantly after FS-LASIK surgery, and this was mainly observed as increases in coma and spherical aberration. The greater the ablation depth, the larger the influence on spherical aberrations. The greater the RCST/CT, the smaller the influence of HOAs on the cornea.

Keywords: Laser-assisted in situ keratomileusis (LASIK); femtosecond; high-order aberration; RCST/CT (residual corneal stroma thickness/corneal thickness)

Submitted Apr 28, 2021. Accepted for publication Jul 20, 2021.

doi: 10.21037/apm-21-1677

View this article at: https://dx.doi.org/10.21037/apm-21-1677

^ ORCID: Ziqing Feng, 0000-0003-1481-2045; Xue Li, 0000-0002-8456-2910. 


\section{Introduction}

Femtosecond laser-assisted in situ keratomileusis (FSLASIK) has gained widespread acceptance over the past decade as a mainstream, safe, stable, predictable, and effective treatment for the correction of myopia and myopic astigmatism (1-3). The corneal flap produced by the femtosecond laser is not only suitable for a wide range of people, and has high safety, but also the thickness of the flap is uniform, the ablation accuracy is good, and the corneal fiber is less damaged. However, it is associated with certain complications such as glare, halos, diplopia, and night vision disturbance. It has been reported that these symptoms are always related to high-order aberrations (HOAs) induced after surgery (4-7).

Previous studies have suggested that corneal flap, ablation diameter, and ablation depth can induce aberrations $(8,9)$. Wang et al. have reported that FS-LASIK changes in corneal aberration occurred mainly on the anterior surface, have a significant effect on visual quality (10). Ablation depth can change the corneal shape and biomechanical responses, both of which are significantly correlated with increases in total HOAs $(11,12)$, and myopia could ablation more tissue. However, ablation depth alone does not fully reflect the impact of refractive surgery on corneal biomechanics, corneal morphology, and postoperative aberration. Therefore, this study used RCST/CT (13) (residual corneal stroma thickness/corneal thickness) to investigate HOA changes after FS-LASIK in myopia patients. We present the following article in accordance with the STROBE reporting checklist (available at https:// dx.doi.org/10.21037/apm-21-1677).

\section{Methods}

\section{Patients}

A total of 65 eyes from 39 patients with high myopia, who had FS-LASIK performed in the Center of Optometry of the First Affiliated Hospital of Harbin Medical University during August 2019 to August 2020, was included in this study. The following inclusion criteria were applied to participants: stable refractive errors for at least 2 years (a change of $\pm 0.50 \mathrm{D}$ or less); corrected distance visual acuity (CDVA) of $16 / 20$ or better prior to surgery; preoperative sphere between -6.00 to $-11.00 \mathrm{D}$, and cylinder less than $-2.50 \mathrm{D}$; and the corneal were shape the rules and transparent, with central thickness greater than $500 \mu \mathrm{m}$, and the calculated residual stroma not less than $250 \mu \mathrm{m}$. Patients were excluded from this study if they presented with a history of amblyopia, corneal disease, cataracts, glaucoma, or retinal disease. Patients were instructed to stop wearing soft contact lenses at least 2 weeks prior to surgery. No post-surgical complications were reported and all patients had a postoperative UDVA of 20/20. All procedures performed in this study involving human participants were in accordance with the Declaration of Helsinki (as revised in 2013). The study was approved by the ethics board of the Department of Optometry of the First Affiliated Hospital of Harbin Medical University (No. 201979) and informed consent was taken from all the patients.

\section{Femtosecond laser-assisted laser in-situ keratomileusis}

During FS-LASIK, the cornea was pre-anesthetized before the operation. The suction ring was centered over the pupil, and the flap was created using a Wavelight FS200 femtosecond laser system (Alcon, USA). Flap thickness was $110 \mu \mathrm{m}, 8.5-9.0 \mathrm{~mm}$ transverse and $8-8.5 \mathrm{~mm}$ longitudinal diameter. After the corneal flap was created and lifted, the suction was switched off. A spatula was carefully passed across the flap starting at the hinge and swept inferiorly to lift the flap for excimer laser ablation. The corneal stroma was ablated in a $6.0-6.5 \mathrm{~mm}$ diameter optical zone using an EX500 excimer laser system (Alcon, USA) at a power setting of $500 \mathrm{KHz}$. Finally, the stromal bed was flushed with a balanced salt solution, and the flap was repositioned after ablation.

\section{Postoperative care}

Patients were instructed to wear a clear eye mask after the operation, and prescribed the following medications: $0.1 \%$ sodium hyaluronate eye drops (Santen, Japan) four times a day for 3-4 weeks; anti-infection treatment (levofloxacin eye drops, Santen, Japan) four times a day for 3-4 weeks; and antiinflammatory treatment (fluorometholone eye drops, Santen, Japan) four times daily for 2 days, three times daily for 2 days, two times daily for 2 days, and once a day for 2 days.

\section{Preoperative and postoperative examination}

The preoperative examinations for each patient included the following: uncorrected distance visual acuity (UDVA), near vision, corrected distance visual acuity (CDVA), slitlamp examination, dilated optometry, intraocular pressure (IOP), corneal topography, ocular wavefront analysis, 
Table 1 Demographics and preoperative data of included patients

\begin{tabular}{lcc}
\hline Parameters & Range & Mean \pm SD \\
\hline Age (years) & $18-40$ & $25.78 \pm 0.85$ \\
Sphere refraction (D) & -6.00 to -11.00 & $-8.32 \pm 0.85$ \\
Cylinder refraction (D) & 0 to -2.50 & $-0.54 \pm 0.49$ \\
Spherical equivalent (D) & -6.25 to -11.25 & $-8.59 \pm 0.84$ \\
RCST/CT (\%) & 55.41 to 66.72 & $61.39 \pm 2.45$ \\
Ablation depth $(\mu \mathrm{m})$ & 78 to 125 & $94.12 \pm 8.91$ \\
\hline
\end{tabular}

RCST/CT, residual corneal stroma thickness/corneal thickness.

dilated fundus examination, and Sirius Scheimpflug-Placido topography. Postoperative examinations included UDVA, CDVA, slit-lamp examination, IOP, Sirius ScheimpflugPlacido topography at 1 week, 1 month, and 3 months postoperatively.

\section{Outcome measurements}

Corneal aberrations and central corneal thickness (CCT) were measured by Sirius Scheimpflug-Placido topography (CSO, Florence, Italy) and found to be more than $6 \mathrm{~mm}$ diameter. The quality factor was automatically evaluated, and only the measurements marked as "OK" in quality specification were considered to be valid. The root mean square (RMS) of horizontal coma $\left(Z_{3}{ }^{1}\right)$, horizontal trefoil $\left(Z_{3}^{3}\right)$, spherical aberration $\left(Z_{4}^{0}\right)$, second horizontal coma $\left(Z_{5}^{1}\right)$, second horizontal trefoil $\left(Z_{5}{ }^{3}\right)$, pentafoil $\left(Z_{5}^{5}\right)$, second spherical aberration $\left(Z_{6}^{0}\right)$, and total highorder aberrations (HOAs) from the Sirius were measured before surgery and at 1 week, 1 month, and 3 months postoperatively.

Ablation depth and thickness of the corneal stromal under flap were measured by Allegretto Wavelight EX500 (Alcon, USA) during the surgery, followed by the calculation of the residual corneal stroma thickness (RCST; where RCST is the thickness of corneal stromal under flap minus ablation depth).

\section{Statistical analysis}

Measured outcomes were analyzed using IBM SPSS version 22.0 software. The Kolmogorov-Smirnov test was used to evaluate the normality of the sample. Analysis of variance tests were used for statistical analysis of normally distributed data which were represented as mean \pm standard deviation (SD). One-way repeated measurements analysis of variance was used for the comparison of the differences of Zernike and HOAs before surgery and at 1 week, 1 month, and 3 months post-surgery. The differences between two different follow-up visits were compared with LSD- $t$-tests. The relationship between RCST/CT and HOA changes, and ablation depth and HOA changes were analyzed by the Pearson correlation test. $\mathrm{P}$ values less than 0.05 were considered statistically significant.

\section{Results}

A total of 65 eyes from 39 patients ( 21 male and 18 female) with high myopia, who had FS-LASIK performed in our hospital, was included in this study. The patient demographics and preoperative data are summarized in Table 1.

\section{A comparison of Zernike and high-order aberration root mean square values before and after femtosecond laser- assisted in situ keratomileusis}

Preoperative $Z_{3}{ }^{1}, Z_{4}{ }^{0}, Z_{5}{ }^{1}, Z_{5}{ }^{3}, Z_{5}^{5}, Z_{6}{ }^{0}$, and HOA RMS values were compared with post-FS-LASIK values at 1 week, 1 month, and 3 months. No significant differences were found in $Z_{3}^{3}$ values pre- and post-surgery $(P>0.05$; Table 2, Figure 1). However, $Z_{3}{ }^{1}, Z_{4}{ }^{0}, Z_{5}{ }^{1}, Z_{5}{ }^{3}, Z_{5}{ }^{5}, Z_{6}{ }^{0}$, and HOAs increased 2.17-, 3.96-, 5.33-, 2-, 1.54-, 17.25-, and 2.47fold, respectively, 1 week after surgery compared with preoperative values. At 1 month post-surgery, the $Z_{3}{ }^{1}, Z_{4}{ }^{0}$, $Z_{5}{ }^{1}, Z_{5}{ }^{3}, Z_{5}{ }^{5}, Z_{6}{ }^{0}$, and HOAs increased 2.18-, 3.80-, 5.30, 1.69-, 1.48-, 16.5-, and 2.46-fold, respectively, compared with preoperative values. At 3 months after surgery, the $Z_{3}{ }^{1}$, $Z_{4}^{0}, Z_{5}^{1}, Z_{5}^{3}, Z_{5}^{5}, Z_{6}^{0}$, and HOA values had increased 2.52-, 4.09-, 5.04-, 1.84-, 1.46-, 16.92-, and 2.64-fold, respectively, compared to preoperative values. 
Table 2 A comparison of Zernike and HOA RMS before and after FS-LASIK ( $x \pm s, \mu \mathrm{m})$

\begin{tabular}{|c|c|c|c|c|c|c|}
\hline Aberrations & Pre-operation & 1 week post-operation & 1 month post-operation & 3 months post-operation & $\mathrm{F}$ & $P$ \\
\hline $\mathrm{Z}_{3}{ }^{1}$ & $0.206 \pm 0.110$ & $0.448 \pm 0.258^{\mathrm{a}}$ & $0.449 \pm 0.273^{\mathrm{a}}$ & $0.519 \pm 0.253^{\mathrm{abc}}$ & 38.341 & $<0.001$ \\
\hline $\mathrm{Z}_{3}^{3}$ & $0.190 \pm 0.083$ & $0.221 \pm 0.090$ & $0.230 \pm 0.134$ & $0.198 \pm 0.111$ & 2.253 & 0.085 \\
\hline $\mathrm{Z}_{4}{ }^{0}$ & $-0.172 \pm 0.079$ & $-0.681 \pm 0.259^{a}$ & $-0.654 \pm 0.258^{\mathrm{ab}}$ & $-0.704 \pm 0.265^{\mathrm{ac}}$ & 146.243 & $<0.001$ \\
\hline$Z_{5}^{1}$ & $0.027 \pm 0.016$ & $0.144 \pm 0.084^{a}$ & $0.143 \pm 0.080^{a}$ & $0.136 \pm 0.068^{a}$ & 51.602 & $<0.001$ \\
\hline$Z_{5}^{5}$ & $0.048 \pm 0.022$ & $0.074 \pm 0.051^{a}$ & $0.071 \pm 0.042^{a}$ & $0.070 \pm 0.047^{a}$ & 3.961 & 0.010 \\
\hline $\mathrm{Z}_{6}^{0}$ & $-0.012 \pm 0.018$ & $-0.207 \pm 0.080^{a}$ & $-0.198 \pm 0.098^{a}$ & $-0.203 \pm 0.077^{\mathrm{a}}$ & 144.835 & $<0.001$ \\
\hline HOAs & $0.385 \pm 0.092$ & $0.952 \pm 0.291^{a}$ & $0.948 \pm 0.283^{\mathrm{a}}$ & $1.016 \pm 0.274^{\mathrm{abc}}$ & 162.333 & $<0.001$ \\
\hline
\end{tabular}

${ }^{\mathrm{a}} \mathrm{P}<0.05$, compared with pre-operation; ${ }^{\mathrm{b}} \mathrm{P}<0.05$, compared with 1 week post-operation; ${ }^{\mathrm{c}} \mathrm{P}<0.05$, compared with 1 month post-operation; statistical analysis performed by repeated measurements analysis of variance and LSD- $t$ tests. HOA, high-order aberration; RMS, root mean square; FS-LASIK, femtosecond laser-assisted in situ keratomileusis.
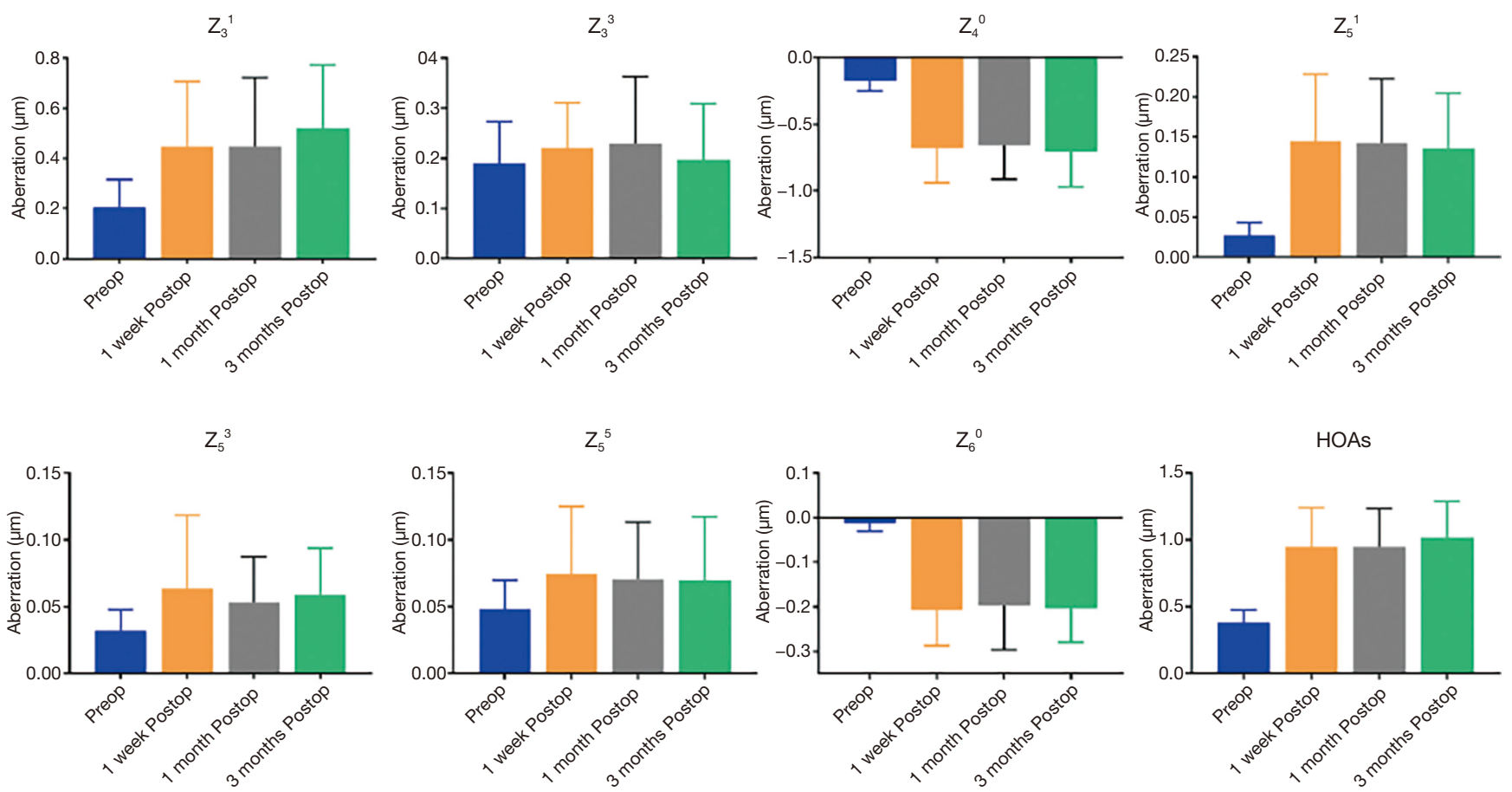

Figure 1 A comparison of $Z_{3}{ }^{1}, Z_{3}{ }^{3}, Z_{4}{ }^{0}, Z_{5}{ }^{1}, Z_{5}{ }^{3}, Z_{5}{ }^{5}, Z_{6}{ }^{0}$, and HOAs before and after FS-LASIK. Preop, pre-operation; postop, post-operation; HOAs, high-order aberrations.

Compared to 1 week after surgery, the $\mathrm{Z}_{4}{ }^{0}$ value significantly decreased at 1 month post-surgery $(\mathrm{P}<0.05)$. $\mathrm{Z}_{3}{ }^{1}$ and HOAs significantly increased at 3 months after surgery compared with the values at 1 week $(\mathrm{P}<0.05)$. $\mathrm{Z}_{3}{ }^{1}, \mathrm{Z}_{4}{ }^{0}$, HOAs were significantly increased at 3 months compared with those at 1 week postoperatively $(\mathrm{P}<0.05)$. $Z_{5}{ }^{1}, Z_{5}{ }^{3}, Z_{5}^{5}$, and $Z_{6}{ }^{0}$ showed no significant differences postoperatively.

A comparison of Pearson correlations between high-order aberration changes and ablation depth before and after FS-LASIK

There was a positive correlations between ablation depth 
Table 3 The correlation coefficient between eye Zernike coefficients, high-order aberration root mean square changes, and ablation depth

\begin{tabular}{|c|c|c|c|c|c|c|}
\hline Aberrations & \multicolumn{2}{|c|}{1 week post-operation $(n=65)$} & \multicolumn{2}{|c|}{1 month post-operation $(n=65)$} & \multicolumn{2}{|c|}{3 months post-operation $(n=43)$} \\
\hline $\mathrm{Z}_{3}{ }^{1}$ & -0.084 & 0.506 & -0.116 & 0.358 & -0.056 & 0.720 \\
\hline $\mathrm{Z}_{3}^{3}$ & -0.189 & 0.132 & -0.075 & 0.553 & -0.139 & 0.374 \\
\hline $\mathrm{Z}_{4}{ }^{0}$ & 0.209 & 0.095 & 0.223 & 0.075 & 0.439 & $0.003^{*}$ \\
\hline $\mathrm{Z}_{5}^{3}$ & -0.211 & 0.092 & 0.017 & 0.892 & -0.094 & 0.550 \\
\hline$Z_{5}^{5}$ & -0.077 & 0.542 & -0.063 & 0.618 & 0.111 & 0.477 \\
\hline $\mathrm{Z}_{6}^{0}$ & 0.291 & $0.019^{\star}$ & 0.337 & $0.006^{\star}$ & 0.336 & $0.027^{*}$ \\
\hline HOAs & 0.101 & 0.421 & 0.081 & 0.521 & 0.252 & 0.104 \\
\hline
\end{tabular}

${ }^{*} \mathrm{P}<0.05$ indicates correlation coefficient $r$ has statistical significance. HOA, high-order aberration.

Table 4 The correlation coefficient between eye Zernike coefficients, HOA RMS changes, and RCST/CT after FS-LASIK

\begin{tabular}{|c|c|c|c|c|c|c|}
\hline Aberrations & \multicolumn{2}{|c|}{1 week post-operation $(n=65)$} & \multicolumn{2}{|c|}{1 month post-operation $(n=65)$} & \multicolumn{2}{|c|}{3 months post-operation $(n=43)$} \\
\hline$Z_{3}^{1}$ & 0.088 & 0.486 & -0.019 & 0.882 & 0.044 & 0.780 \\
\hline $\mathrm{Z}_{3}^{3}$ & 0.241 & 0.053 & 0.047 & 0.708 & -0.147 & 0.346 \\
\hline $\mathrm{Z}_{4}{ }^{0}$ & -0.105 & 0.407 & -0.078 & 0.536 & -0.322 & $0.035^{\star}$ \\
\hline $\mathrm{Z}_{5}^{3}$ & 0.046 & 0.714 & -0.138 & 0.272 & -0.245 & 0.113 \\
\hline$Z_{5}^{5}$ & 0.080 & 0.526 & -0.053 & 0.674 & -0.279 & 0.070 \\
\hline $\mathrm{Z}_{6}^{0}$ & -0.212 & 0.091 & -0.209 & 0.095 & -0.412 & $0.006^{\star}$ \\
\hline HOAs & -0.143 & 0.255 & -0.178 & 0.157 & -0.321 & $0.036^{*}$ \\
\hline
\end{tabular}

${ }^{\star} \mathrm{P}<0.05$, correlation coefficient $r$ has statistical significance. HOA, high-order aberration; RMS, root mean square; RCST/CT, residual corneal stroma thickness/corneal thickness; FS-LASIK, femtosecond laser-assisted in situ keratomileusis.

and $Z_{6}^{0}$ at 1 week and 1 month after surgery $(r=0.291,0.337$, $\mathrm{P}<0.05)$. Ablation depth was also positively correlated with $Z_{4}^{0}$ and $Z_{6}^{0}$ at 3 months after surgery ( $r=0.439,0.336$, $\mathrm{P}<0.05)$. No correlations were found between other Zernike terms $(\mathrm{P}>0.05$; Table 3).

\section{A comparison of Pearson correlations between high-order aberration changes and RCST/CT before and after FS-LASIK}

The RCST/CT was negatively correlated with $Z_{4}^{0}, Z_{6}^{0}$, and HOAs at 3 months after surgery $(\mathrm{r}=-0.322,-0.412$, -0.321 , respectively; $\mathrm{P}<0.05)$. However, RCST/CT was not significantly correlated with Zernike coefficients and HOAs and at 1 week and 1 month after surgery (Table 4).

\section{Discussion}

In this study, most patients gained satisfactory visual and stability outcomes after FS-LASIK surgery. However, some patients complained of visual disturbances such as double vision, glare, halos, starbursts, and a decrease in night vision quality, which can be induced by HOAs in the cornea. Corneal stroma ablation can change the corneal asphericity and biomechanics, which may account for the variations in aberrations.

In addition, the higher the diopter, the more corneal tissue will be ablation and the number of people with high 
myopia is increasing year by year and high prevalence of high myopia in young adults (10-20\%) (14). Therefore, this study investigated the time-dependent changes of HOAs based on RCST/CT (residual corneal stroma thickness/ corneal thickness) after FS-LASIK in patients with high myopia.

An aberration is the difference between an ideal image and a real image and can be divided into low-order aberrations and high-order aberrations. Zernike is usually used to express ocular wavefront errors and a root mean square (RMS) is used to measure the magnitude of the wavefront aberration. HOAs can have a great influence on visual quality (15), and hence third order aberrations (S3-S6) and total highorder aberrations (Sh) were measured. Khan et al. (16) reported that among Zernike coefficients, coma, spherical and HOAs significantly increased 1.86-, 2.44-, and 1.56-fold, respectively, at 1 month after FS-LASIK, suggesting obvious spherical aberration. Wang et al. (10) reported that HOAs on the whole and anterior corneal surfaces increased significantly at 1 month after surgery, and the spherical aberration on the whole corneal and anterior corneal surfaces were significantly increased at 1 and 3 months postoperatively, along with the vertical coma on the whole and anterior corneal surfaces at 1 and 3 months. However, there was no significant difference in horizontal coma or trefoil after surgery. In the report by Vega-Estrada (12), coma, spherical, and HOAs increased at 3 months after FS-LASIK, but trefoil remained unchanged. In this current study, $Z_{3}{ }^{1}, Z_{4}{ }^{0}, Z_{5}{ }^{1}, Z_{5}{ }^{3}, Z_{5}{ }^{5}, Z_{6}{ }^{0}$, and HOAs were significantly increased at 1 week, 1 month, and 3 months after surgery. The variations of spherical aberrations were greater than variations in coma, and there was no significant decrease at 3 months, which was in agreement with previous studies. However, there was no obvious relationship between postoperative time points and changes in HOAs.

Some reports have suggested that coma and spherical aberrations play a major role in affecting vision quality and showed a correlation between starburst and spherical aberrations, glare and spherical aberrations, with as well as a correlation between diplopia and horizontal coma $(17,18)$. Coma can be caused by the corneal flap, an unsmooth corneal stromal bed, as well as the corneal wound-healing response (19-21). Previous studies have suggested that postoperative spherical aberrations may be caused by the "cosine effect", and that ablation changes the corneal shape from long oval to flat oval after surgery (22).

$\mathrm{Hu}$ et al. (23) demonstrated a significant correlation between induced $Z_{1}^{2}$ and $Z_{2}^{4}$ (spherical aberrations) and ablation depth. The results of our current study found positive correlations between ablation depth and $\mathrm{Z}_{6}^{0}$ at 1 week and 1 month after surgery, as well as between ablation depth and $Z_{4}{ }^{0}$ and $Z_{6}{ }^{0}$ at 3 months after surgery. Spherical aberration means the asphericity of the cornea. The central curvature of the cornea is higher than the periphery, the corneal shape changes to flat oval after central cornea ablation, and the peripheral light is focused in front of the focus of the paraxial. Therefore, the spherical aberration shifts to a negative value. The more central the corneal ablation, the greater will be the change in spherical aberration. In addition, the variation of spherical aberration is also related to the size of the ablation zone. In this study, the ablation zone diameter of all the patients was $6.0-7.0 \mathrm{~mm}$, and $6.0 \mathrm{~mm}$ was selected for the measurement of HOAs, thereby enabling a good comparison of the relationship between the changes in HOAs and ablation depth.

Changes in corneal shape can lead to changes in biomechanics, and thus, HOAs may be related to biomechanics FS-LASIK surgery. Li and colleagues (13) suggested that RCST/CT can reflect not only the influence of the residual stromal thickness on the cornea after surgery, but also the original morphology of the cornea before surgery. This allows accurate reflection of the influence of corneal refractive surgery on corneal biomechanics. In addition, posterior corneal surface anterior protrusion was found at 3 months after LASIK, and it was negatively correlated to RCST/CT. In a later study (24), the same authors showed that the greater the RCST/CT, the less the impact on corneal biomechanics after FS-LASIK. Furthermore, Ji and colleagues (25) found a positive correlation between posterior corneal surface anterior protrusion and induced HOAs.

In this current investigation, RCST/CT was negatively correlated with $\mathrm{Z}_{4}{ }^{0}, \mathrm{Z}_{6}{ }^{0}$, and HOAs at 3 months after surgery and no significant correlations were found between Zernike coefficients terms of high-order aberration, HOAs, and RCST/CT at 1 week and 1 month after surgery. In terms of corneal biomechanical mechanisms, ablation of the corneal stroma would decrease the elastic strength of the cornea against intraocular pressure, which would lead to posterior corneal surface anterior protrusion. As corneal biomechanical stability is maintained largely by the thickness of the residual corneal stroma after surgery, the greater the RCST/CT, the more the corneal biomechanical stability and the less the changes in aberrations. Consistent with previous studies (13), at 3 months post-surgery, RCST/CT affected more Zernike coefficients compared 
with 1 week and 1 month post-surgery, suggesting that the morphological changes of the cornea were most significant at 3 months after LASIK and stabilized or gradually recovered by 6 months.

This study also demonstrated that RCST/CT influenced spherical aberration changes, which confirmed that RCST/CT affects asphericity after surgery. Retaining greater RCST/CT may induce less spherical aberrations. Therefore, by designing the RCST/CT of the cornea before surgery, HOAs may be reduced, thereby improving postoperative visual quality and satisfaction for patients.

Although this report examined the relationship between HOA changes and RCST/CT, a specific value for each spherical equivalent could not be determined and thus it was not possible to build a prediction model for postoperative visual quality. Further studies should include more subjects with different diopters.

\section{Conclusions}

More corneal HOAs in high myopia patients increased significantly after FS-LASIK, which was mainly observed as increases in coma and spherical aberrations. Furthermore, the greater the ablation depth, the larger the influence on spherical aberrations, and the greater the RCST/CT, the smaller the influence on HOAs of the cornea.

\section{Acknowledgments}

The authors wish to thank the Center of Optometry of the First Affiliated Hospital of Harbin Medical University, Harbin, China.

Funding: None.

\section{Footnote}

Reporting Checklist: The authors have completed the STROBE reporting checklist. Available at https://dx.doi. org/10.21037/apm-21-1677

Data Sharing Statement: Available at https://dx.doi. org/10.21037/apm-21-1677

Conflicts of Interest: All authors have completed the ICMJE uniform disclosure form (available at https://dx.doi. org/10.21037/apm-21-1677). The authors have no conflicts of interest to declare.
Ethical Statement: The authors are accountable for all aspects of the work in ensuring that questions related to the accuracy or integrity of any part of the work are appropriately investigated and resolved. All procedures performed in this study involving human participants were in accordance with the Declaration of Helsinki (as revised in 2013). The study was approved by the ethics board of the Department of Optometry of the First Affiliated Hospital of Harbin Medical University (No. 201979) and informed consent was taken from all the patients.

Open Access Statement: This is an Open Access article distributed in accordance with the Creative Commons Attribution-NonCommercial-NoDerivs 4.0 International License (CC BY-NC-ND 4.0), which permits the noncommercial replication and distribution of the article with the strict proviso that no changes or edits are made and the original work is properly cited (including links to both the formal publication through the relevant DOI and the license). See: https://creativecommons.org/licenses/by-nc-nd/4.0/.

\section{References}

1. MacRae S. Thin-flap femtosecond LASIK. J Refract Surg 2010;26:469-70; author reply 470.

2. Wang XZ, Cui R, Song XD, et al. Comparison of the accuracy of intraocular lens power calculation formulas for eyes after corneal refractive surgery. Ann Transl Med 2020;8:871.

3. Arora R, Goel Y, Goyal JL, et al. Refractive outcome of wavefront guided laser in situ keratomileusis and wavefront guided photorefractive keratectomy in high preexisting higher order aberration. Cont Lens Anterior Eye 2015;38:127-33.

4. Yap EY, Kowal L. Diplopia as a complication of laser in situ keratomileusis surgery. Clin Exp Ophthalmol 2001;29:268-71.

5. Oliveira CM, Ferreira A, Franco S. Wavefront analysis and Zernike polynomial decomposition for evaluation of corneal optical quality. J Cataract Refract Surg 2012;38:343-56.

6. Mrochen M, Kaemmerer M, Mierdel P, et al. Increased higher-order optical aberrations after laser refractive surgery: a problem of subclinical decentration. J Cataract Refract Surg 2001;27:362-9.

7. Wang $\mathrm{Y}$, Zhao K, Yang $\mathrm{X}$, et al. Higher order aberrations and low contrast vision function in myopic eyes $(-3.00$ to $-6.00 \mathrm{~d}$ ) under mesopic conditions. J Refract Surg 
2011;27:127-34.

8. Porter J, MacRae S, Yoon G, et al. Separate effects of the microkeratome incision and laser ablation on the eye's wave aberration. Am J Ophthalmol 2003;136:327-37.

9. Ganesh S, Brar S, Arra RR. Refractive lenticule extraction small incision lenticule extraction: A new refractive surgery paradigm. Indian J Ophthalmol 2018;66:10-9.

10. Wang J, Ren Y, Liang K, et al. Changes of corneal highorder aberrations after femtosecond laser-assisted in situ keratomileusis. Medicine (Baltimore) 2018;97:e0618.

11. Li K, Zhang CW, Hong DJ, et al. Clinical study on combining femtosecond thin- flap and LASIK with the Triple-A profile for high myopia correction. BMC Ophthalmol 2019;19:107.

12. Vega-Estrada A, Alió JL, Arba Mosquera S, et al. Corneal higher order aberrations after LASIK for high myopia with a fast repetition rate excimer laser, optimized ablation profile, and femtosecond laser-assisted flap. J Refract Surg 2012;28:689-96.

13. Li X, Zhao YJ, Zhang L, et al. Comparison of posterior corneal surface after LASIK and LASEK in myopia. Recent Advances in Ophthalmology 2012;32:545-7.

14. Morgan IG, French AN, Ashby RS, et al. The epidemics of myopia: Aetiology and prevention. Prog Retin Eye Res 2018;62:134-49.

15. Zhao PF, Li SM, Lu J, et al. Effects of higher-order aberrations on contrast sensitivity in normal eyes of a large myopic population. Int J Ophthalmol 2017;10:1407-11.

16. Khan MS, Humayun S, Fawad A, et al. Effect of wavefront optimized LASIK on higher order aberrations in myopic patients. Pak J Med Sci 2015;31:1223-6.

17. Li X, Wang Y, Dou R. Aberration compensation between anterior and posterior corneal surfaces after Small incision lenticule extraction and Femtosecond laser-assisted laser in-situ keratomileusis. Ophthalmic Physiol Opt

Cite this article as: Feng Z, Wang Q, Du C, Yang F, Li X. High-order aberration changes after femtosecond LASIK surgery in patients with high myopia. Ann Palliat Med 2021;10(7):7689-7696. doi: 10.21037/apm-21-1677
2015;35:540-51.

18. Piao J, Li YJ, Whang WJ, et al. Comparative evaluation of visual outcomes and corneal asphericity after laser-assisted in situ keratomileusis with the six-dimension Amaris excimer laser system. PLoS One 2017;12:e0171851.

19. Jin HY, Wan T, Wu F, et al. Comparison of visual results and higher-order aberrations after small incision lenticule extraction (SMILE): high myopia vs. mild to moderate myopia. BMC Ophthalmol 2017;17:118.

20. Pallikaris IG, Kymionis GD, Panagopoulou SI, et al. Induced optical aberrations following formation of a laser in situ keratomileusis flap. J Cataract Refract Surg 2002;28:1737-41.

21. Liu TX, Chen YT, Dan TT, et al. Four-year followup of corneal aberrations and visual functions of myopic patients after laser in situ keratomileusis. Pak J Med Sci 2015;31:1453-6.

22. Wang Y, Zhao KX. High order aberration and visual quality after corneal refractive surgery. Chinese Journal of Ophthalmologic 2011;47:664-8.

23. $\mathrm{Hu} \mathrm{L}, \mathrm{Yu} \mathrm{Y}, \mathrm{Wu}$ JX, et al. Wavefront aberration and its association with central corneal thickness and ablation depth in myopic eyes after LASIK surgery. Chinese Journal of Optometry Ophthalmology and Visual Science 2010;12:357-62.

24. Li ZJ, Li X, Du CY, et al. Comparison of corneaI biomechanicaI changes at earIy stage after femtosecond LASIK and microkeratome LASIK. International Eye Science 2015;(3):428-31.

25. Ji P, Bi HS, Wang XR, et al. Multiplicity of high order aberration changes after LASIK for myopia and astigmatism. Recent Advances in Ophthalmology 2014;34:352-4.

(English Language Editor: J. Teoh) 